\title{
Realisation of the metre by using a femtosecond laser frequency comb: applications in optical frequency metrology
}

\author{
Bousselham Samoudi* \\ National School of Applied Sciences of Safi, Cadi Ayyad University, Sidi Bouzid's Road, BP 63, Safi 46000, Morocco
}

Received: 18 September 2016 / Accepted: 2 May 2017

\begin{abstract}
The appearance of the frequency comb technology, awarded the Nobel Prize in Physics 2005, has enormously revolutionized the metrology of optical frequencies, eliminating the need for complicated frequency chains. By direct linking to the unit of time, the second, through frequency standards $(C s, R b)$, by using femtosecond mode-locked lasers and frequency comb technology, the Spanish Centre of Metrology (CEM) has established a new way of practical realisation of the National Standard of Length, the metre. By stabilising and characterising two free parameters - the repetition frequency $f_{r}$ and the offset frequency $f_{0}$, the frequency comb generator thereby was successfully put into operation. After such realization, the accuracy of the length unit will be increased in two orders of magnitude, that is $2 \times 10^{-13}$ instead of $2.1 \times 10^{-11}$. In this paper we present the results of applying comb generator to the absolute measurement of the three Zeeman stabilized He-Ne lasers operating at $633 \mathrm{~nm}$ with a nominal frequency of $473.612 \mathrm{THz}$. A comparison of these results with those obtained by the current system based on standard iodine stabilized lasers is in good compatibility. A treatment for the evaluation of measurement uncertainty of laser frequency in calibration using a comb in accordance with Guide of Uncertainty Measurement ISO/BIPM is also presented.
\end{abstract}

Keywords: frequency combs / length standard / uncertainty / optical frequency metrology / mode-locked laser

\section{Introduction}

The definition of the unit of length and its practical realisation are based on the adopted value of the speed of light, $c_{0}=299792458 \mathrm{~m} / \mathrm{s}$, and the frequency of an optical transition. Thus, length measurements are intrinsically related to the unit of time. It was adopted by the 17th Conference Générale des Poids et Mesures in 1983 [1]. At the same time, the Comité International des Poids et Mesures (CIPM) made recommendations for the practical realization of the metre, referred to as the mise en pratique (in later text referenced as $\mathrm{MeP}$ ) of the definition. The metre should be realized by one of the following methods [2]:

(a) by means of the length $l$ of the path travelled in vacuum by a plane electromagnetic wave in a time $t$; this length is obtained from the measured time $t$, using the relation $l=c_{0} \cdot t$ and the value of the speed of light in vacuum $c_{0}=299792458 \mathrm{~m} / \mathrm{s}$

(b) by means of the wavelength in vacuum $\lambda$ of a plane electromagnetic wave of frequency $f$; this wavelength is obtained from the measured frequency $f$ using the relation $\lambda=c_{0} / f$ and the value of the speed of light in vacuum $c_{0}=299792458 \mathrm{~m} / \mathrm{s}$;

\footnotetext{
* Corresponding author: b.samoudi@uca.ma
}

(c) by means of one of the radiations from a given list, whose stated wavelength in vacuum or whose stated frequency can be used with the uncertainty shown, provided that the given specifications and accepted good practice are followed.

Various sources of radiation have been recommended as standards of wavelength and have been updated by the CIPM over time $[2,3]$. One of the most important recommended radiations in the field of length metrology and worldwide precision measurements is that at $474 \mathrm{THz}$ $(633 \mathrm{~nm})$ from a He-Ne laser, stabilized on an absorbing hyperfine component in ${ }^{127} \mathrm{I}_{2}$. This laser is used in many laboratories around the world as a practical means of realizing the SI metre and is commonly used for calibrating the frequency of lasers employed in length measurement and precision measurement in atomic physics. The lasers standards work according to method (c) of the Comité consultatif de Longueurs (CCL)/CIPM recommendation for the realization of SI of the metre definition.

To check the values of the standards and the possible improvement of uncertainty, one needs to absolutely measure laser frequency (hundreds of terahertz) relative to caesium clocks (realizing SI definition of the second). This measurement (method $\mathrm{b}$ in $\mathrm{MeP}$ ) was made easier by the invention of optical frequency comb technique [4-6] awarded the Nobel Prize in Physics 2005. 
With this system (frequency comb), the Spanish Centre of Metrology (CEM) is establishing a new practical realization of the metre improved accuracy in two orders of magnitude (i.e. $2 \times 10^{-13}$ instead of $2.1 \times 10^{-11}$ ) with respect to the current system based on iodine stabilized lasers [7,8].

In this paper, the state of art of femtosecond optical frequency comb, including a theoretical background study, is briefly described. The absolute frequency measurement of Zeeman-stabilized He-Ne lasers operating at $633 \mathrm{~nm}$ by the frequency comb and the comparison of the results obtained by the current method of beating frequency, using a standard iodine stabilized lasers (CEM2) have been reported. In both cases, a stability study of the signal beat frequency is presented. Finally, we estimate the measurement uncertainty for comb and laser frequency under calibration using the Guide of Uncertainty Measurement (GUM) ISO/BIPM.

\section{Theoretical background}

Optical frequency combs are routinely used to generate optical radiation with a frequency uncertainty limited only by the uncertainty of the master clock that sets the repetition frequency of the combs. These frequency combs, working in mode-lock regime, have to be designed so that the round trip time for all longitudinal modes is the same. To understand the mode structure of a femtosecond frequency comb and the techniques applied for its stabilization one can look at the idealized case of a pulse circulating in a laser cavity with length $L$ and carrier frequency $f_{c}$ as shown in Figure 1. The output of this laser is a sequence of pulses that are essentially copies of the same pulse separated by the round trip time given by:

$$
T=2 L / v_{g}
$$

where $v_{g}$ is the cavity mean group velocity defined by the round trip time and the cavity length.

The pulses however are not quite identical. This is because the pulse envelope $A(t)$ propagates with $v_{g}$ while the carrier wave travels with its phase velocity. As a result, the carrier shifts with respect to the pulse envelope after each round trip by a phase angle $\Delta \phi$ as shown in Figure 1. The shape of the pulse train in time domain is related to the amplitude and phase spectra by complex Fourier transform.

Let us describe the time evolution of the electric field at one point in space by carrier frequency $f_{c}$ and periodic amplitude/envelope $A$. Unlike the envelope function, which provides us with a more rigorous definition of the pulse repetition time $f_{r}=1 / T$ by demanding $A(t)=A$ $(t+T)$, the electric field is, in general, not expected to be periodic in time. If the periodicity of the envelope function is assumed, the electric field at a given place outside the laser resonator can be written as,

$$
E(t)=\operatorname{Re}\left[A(t) \exp \left(-i 2 \pi f_{c}\right) t\right]
$$

Because the envelope is periodic

$$
E(t)=\operatorname{Re}\left[\sum_{n} A_{n} \exp \left(-i 2 \pi\left(f_{c}+n f_{r}\right) t\right]\right.
$$

where $A_{n}$ are Fourier components of $A(t)$. This equation shows that, under the assumption of a periodic pulse envelope, the resulting spectrum consists of a comb of laser modes that are separated by the pulse repetition frequency. Since $f_{c}$ is not necessarily an integer multiple of $f_{r}$, the modes are shifted with respect to the exact harmonics of the repetition frequency by an offset which can be selected in such a way that $f_{0}<f_{r}$ simply by renumbering the modes:

$$
f_{n}=f_{0}+n f_{r}
$$

where $n$ is a large integer of order $10^{6}$ that indexes the comb line. This equation maps two radio frequencies (RF) $f_{r}$ and $f_{0}$ onto the optical frequencies $f_{n}$. Although the detection of $f_{r}$ is rather effortless and usually lies between a few ten $\mathrm{MHz}$ and a few $\mathrm{GHz}$ depending on the length of the laser resonator, the determination of $f_{0}$ is far more challenging to access unless the frequency comb contains more than an optical octave. $f_{0}$ then is detected by the selfreferencing technique (Fig. 2) with an $f-2 f$ interferometer setup $[9,10]$. Therefore, a mode, with mode number $n$ at the red wing of the comb, whose frequency is given according to equation (4), is frequency doubled in a nonlinear crystal. If the frequency comb covers a full optical octave, a mode with the number $2 n$ should oscillate simultaneously at

$$
f_{2 n}=f_{0}+2 n f_{r}
$$

The beat note between the frequency doubled mode and the mode at $2 n$ yields the offset frequency

$$
2\left(f_{0}+n f_{r}\right)-\left(f_{0}+2 n f_{r}\right)=f_{0}
$$

The frequency of a laser under calibration (continuous wave), $f_{c w}$, is determined by observing a beat frequency, $f_{\text {beat }}$, with the nearest mode of the frequency comb system. Therefore, the $f_{c w}$ is expressed as

$$
f_{c w}=n f_{r} \pm f_{0} \pm f_{\text {beat }} .
$$

The signs of the equation (5) depend on the measurement conditions and can be determined by changing $f_{0}$ and $f_{r}$ while monitoring the resulting change in the beat signal.

If the visible $c w$ lasers are measured after second harmonic generation (SHG), that is our case, equation (5) has to be modified to take the SHG process into consideration. The $f_{0}$ has to be multiplied by a factor of two:

$$
f_{c w}=n f_{r} \pm 2 f_{0} \pm f_{\text {beat }} .
$$

The repetition rate remains unchanged due to the fact that the dominant process in the SHG is sum frequency generation. 


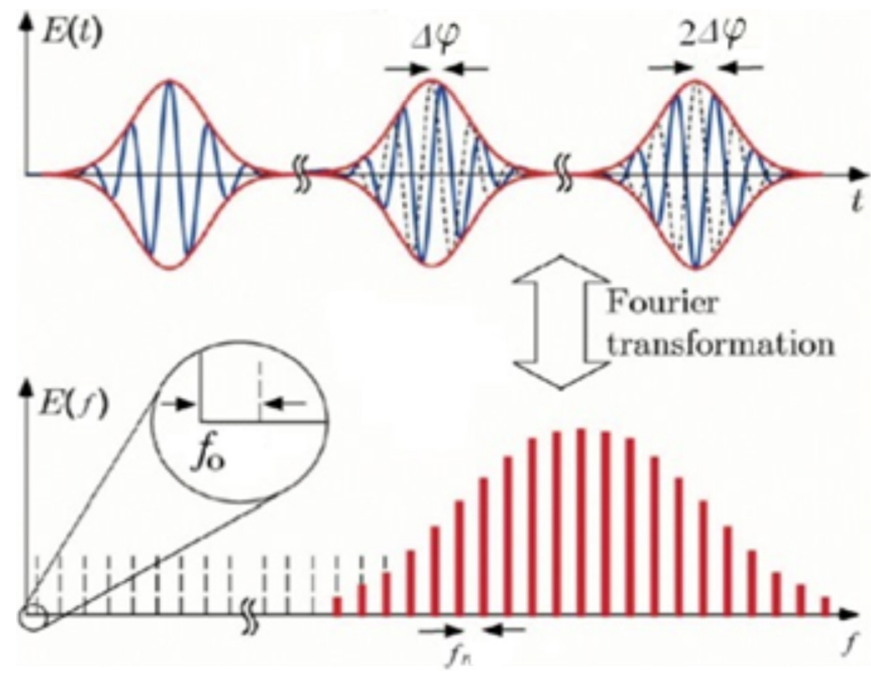

Fig. 1. Representation of the output field of a mode-locked laser in domain time and the corresponding spectrum.

\section{Experimental setup}

The details on experimental arrangements of the femtosecond laser frequency comb were published elsewhere [7,8]. In this paper, a brief summary is presented. The experimental arrangement employed is sketched in Figure 3 . The version of the femtosecond laser frequency comb, the FC1500, developed by Menlo Systems GmbH, is based on a $250 \mathrm{MHz}$ repetition rate mode-locked erbium fibre-ring laser and a non-linear microstructured fibre.

The heart of the frequency comb generator is a fibre laser head with internal erbium doped fibre amplifier (EDFA) for generating high power IR light at $1500 \mathrm{~nm}$ with power up to $2 \mathrm{~mW}$. The femtosecond laser output power is split into two branches and fed to the monitor port and the external parts of the system. One branch is amplified in an external EDFA and spectrally broadened in a highly nonlinear fibre to cover a spectrum of one octave in frequency space, and which will later be doubled in frequency. The beat of the offset frequency $f_{0}$ is detected by means of a nonlinear interferometer. The other branch for generating high power IR light at $1560 \mathrm{~nm}$ for subsequent frequency doubling at $780 \mathrm{~nm}$ radiation, and a photonic crystal fibre setup for subsequent broadening of the SHG output to (530-1000) $\mathrm{nm}$ with power up to $60 \mathrm{~mW}$.

We use a fast p-i-n junction (PIN) diode to detect the offset frequency.

The laser head features an internal fast PIN photo diode to detect the 4th harmonic of the repetition frequency which increases the phase sensitivity.

Two phase locked loops to lock the repetition rate and offset frequency in RF standard allow to transfer the accuracy and stability between the RF and optical domain and are part of the electronics. Frequency counters without dead time are used to measure beat signals with $c w$ lasers and to control the phase locked loops for cycle slips. An oscilloscope and a spectrum analyzer are also included for monitoring purposes.

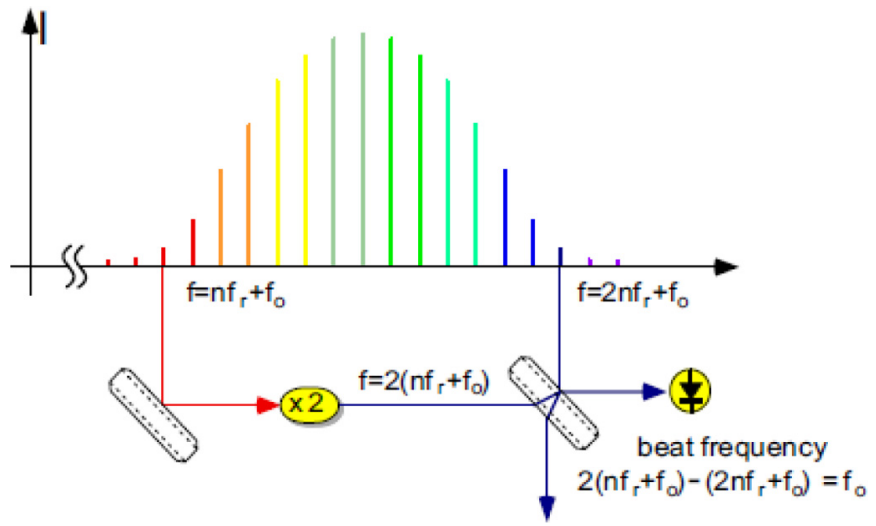

Fig. 2. The self-referencing technique of the optical frequency comb used for the measurement of the carrier-envelope-offset frequency.

The beat detection unit (Fig. 4) consists of a series of silver/gold coated mirrors, polarized beam splitters, $\lambda / 2$ or $\lambda / 4$ waveplates, which aim to place the beams from both the laser comb and calibration in the same polarisation plane, and lead them to a photodetector.

The repetition rate frequency, $f_{r}$, and the carrierenvelope offset frequency $(20 \mathrm{MHz}), f_{0}$, and $f_{\text {beat }}$ were referenced to a primary frequency standard, a $C s$ atomic clock (Symmetricom, model 5071A), integrated into the network of atomic clocks of the Navy Observatory, ROA, in charge of maintaining the Spanish time and frequency standards.

To measure the beat note between Zeeman-stabilized $\mathrm{He}-\mathrm{Ne}$ lasers emitting at a nominal wavelength of $633 \mathrm{~nm}$ and the frequency comb mode, a grating (2100 lines/mm) for spectral filtering was used. The primary difficulties with measuring $f_{\text {beat }}$ arise from the fact that only a very small fraction signal of the comb power is in the mode that gives rise to a beat signal. Several strategies are needed to maximize signal to noise relation and reduce detector saturation effects. Probably the most important single step is simply to take great care in aligning the beam from the test laser with the beam from the comb. To be more precise, what is ideally needed is perfect matching of the wavefronts - two coaxial Gaussian beams with the same waist position and same size, travelling in the same direction. It is very important to assure that the beams overlap very well, travel accurately in the same direction and have similar waist size to obtain a good beat signal. Such beat note frequency was detected by an avalanche photodetector APD210. All relevant frequencies in the experiment have been analyzed and counted by a spectrum analyzer (Hameg, HM5510 model) and frequency counters (Menlo Systems, FXM50 model), respectively, which have been referenced to a primary frequency standard (Symmetricom, 5071A model) with a relative stability of $1 \times 10^{-12}$ at short time, which is worse than real uncertainty of combs and reference RF signals. However, this stability value is definitely sufficient for length measurements. Therefore, the stability of our system is predetermined by the reference source. Currently, to achieve long-term stability it is possible to use H-Masers and fountains based on cold 


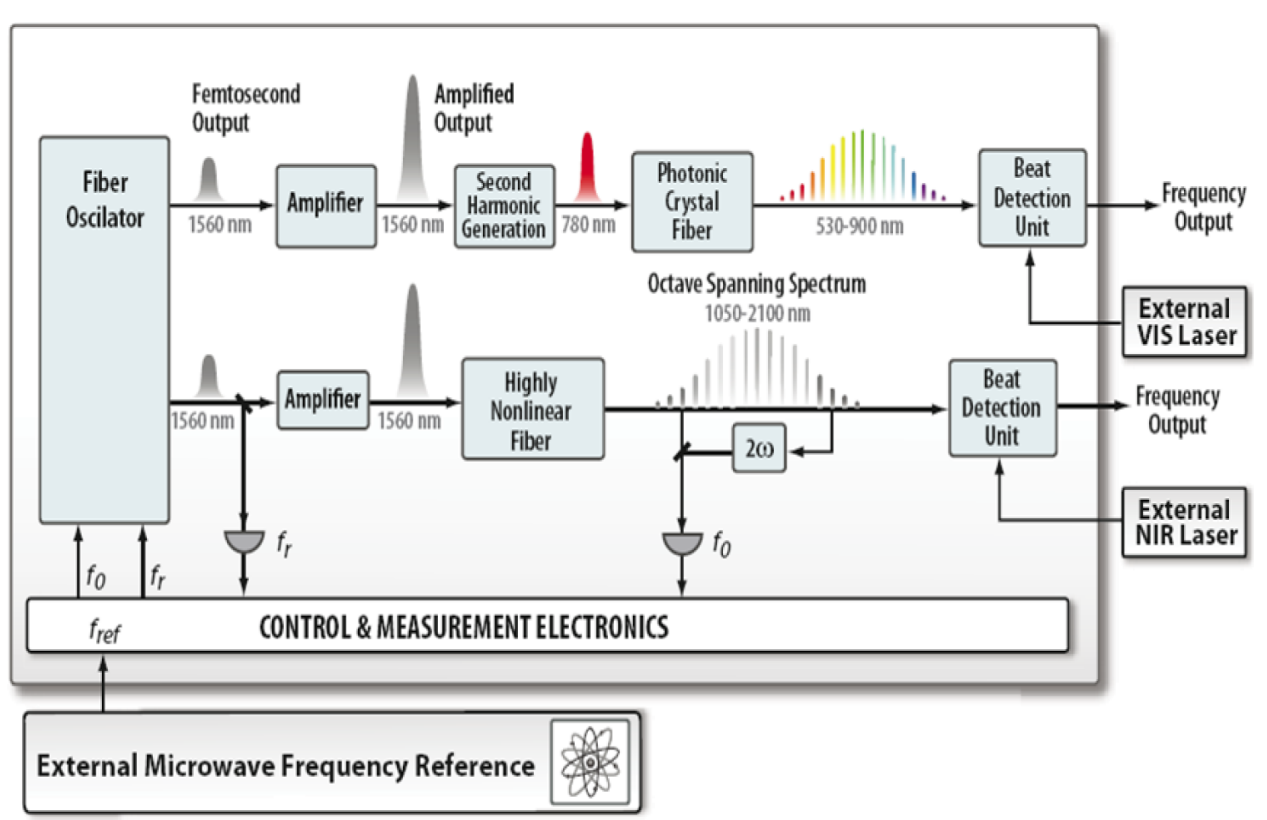

Fig. 3. Experimental setup used to measure the absolute frequency of different Zeeman-stabilized He-Ne lasers operating at $633 \mathrm{~nm}$ with a femtosecond comb generator.

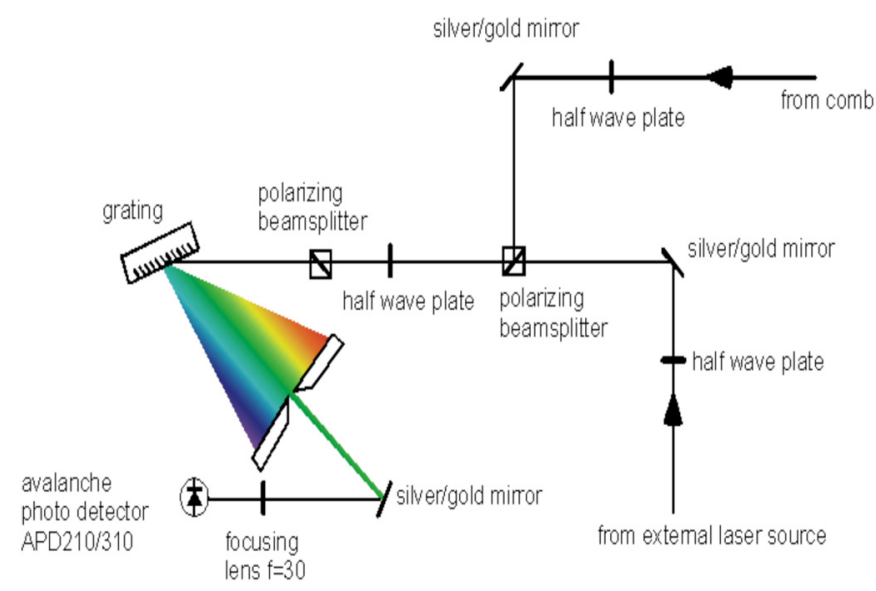

Fig. 4. Beat detection unit used for to measure the absolute frequency of different Zeeman-stabilized He-Ne lasers operating at $633 \mathrm{~nm}$ with a femtosecond comb generator.

atoms. In our case, we are using both $C s$ clock and $R b$ clock disciplined to the previous one, thus ensuring long and short term stability.

During the process of measurement, the frequency comb system as well as the remaining phase is very reliably locked. The frequency counters used for these measurements count continuously every second without dead time for more than $5 \mathrm{~h}$. In our case, the $S / N$ ratio over $30 \mathrm{~dB}$ is sufficient for counting without cycle slips.

\section{Results and discussions}

At CEM we count with several Zeeman-stabilized He-Ne lasers operating at $633 \mathrm{~nm}$. These lasers are traceable to the primary standard (CEM2) He-Ne laser stabilized to hyperfine spectral components of the transition 11-5 R (127) of ${ }^{127} \mathrm{I}_{2}$ vapour in internal cell with a nominal wavelength at $633 \mathrm{~nm}$ with third harmonic locking technique [8]. In this section we present results of absolute frequency measurement of three Zeeman stabilized $\mathrm{He}-\mathrm{Ne}$ laser at $633 \mathrm{~nm}$, using a frequency comb. A comparison of these results with those obtained with primary standard CEM2 laser has been treated.

The $f_{\text {beat }}$ of Zeeman-stabilized He-Ne lasers deviation measurements from the mean value are shown in Figures 5 and 6 . It can be seen that the standard deviations of these measurements are in agreement with the acceptance and rejection criteria established for obtaining reliable results.

The stability of the measured absolute frequency of our wavelength standard is limited by the stability of the reference $C s$ clock and $R b$ clock disciplined to the previous one, for ensuring long and short term stability.

In previous study we calculated the Allan deviation (square root of the Allan variance) the stability of the femtosecond comb reaches $8.38 \times 10^{-11}$ at $1 \mathrm{~s}$ averaging and improves to $3.54 \times 10^{-15}$ after $10000 \mathrm{~s}$ [8]. Figures 7 and 8 show the Allan deviation of frequency beat obtained with the comb system. The beat note and the Allan deviation of HP5519A laser look like the frequency beat and Allan deviation of HP5517D.

It can be seen that Allan deviation of beat frequency obtained with the Tesa laser against the frequency comb describes the typical behaviour with white noise common in the caesium and rubidium time/frequency standards $[11,12]$. However, the stability of HP5517D and HP5519A lasers (Fig. 8) at the longer averaging times is degraded. In particular, the stability plot has a $\tau[11,12]$ slope (time $=\tau$ ) beyond $250 \mathrm{~s}$ that corresponds to the drift (i.e., about $5.6 \times 10^{-9}$ at $5000 \mathrm{~s}$ ). 


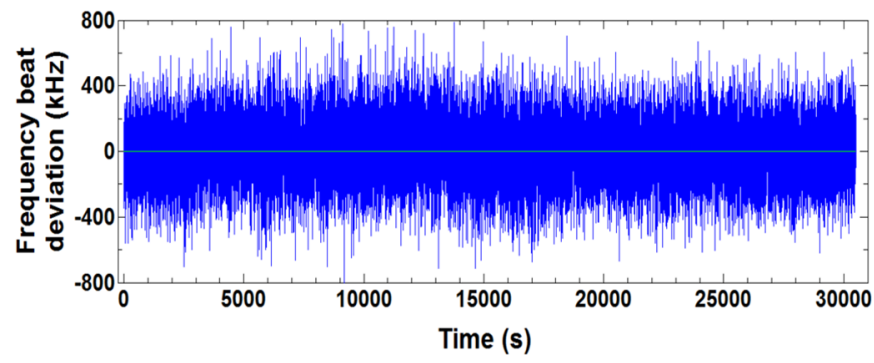

Fig. 5. $f_{\text {beat }}$ deviation of Tesa RSD/SR laser against frequency comb.

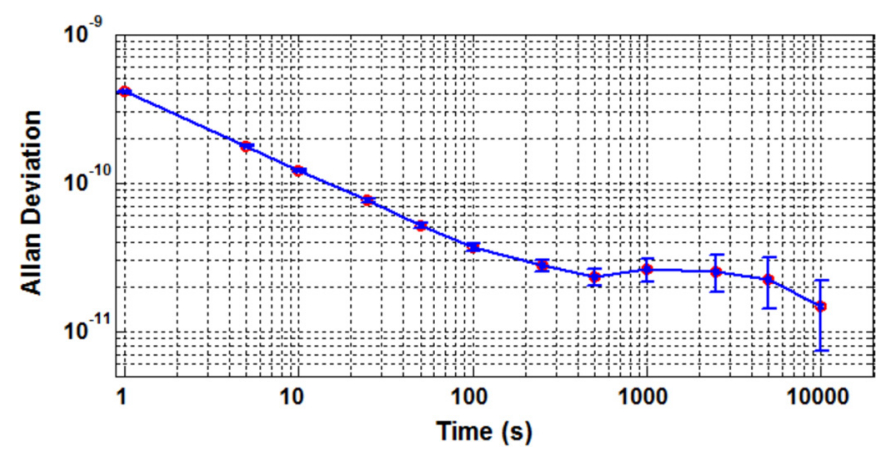

Fig. 7. Allan deviation of $f_{\text {beat }}$ between Tesa RSD/SR and femtosecond comb. Error bars corresponding to the standard uncertainty are shown for longer averaging times, where the error is large enough to be significant.

Evaluating the compatibility coefficient $C$, using the formula

$$
C=\left|X_{1}-X_{2}\right| / \sqrt{U_{1}^{2}+U_{2}^{2}},
$$

where $X_{1}$ and $X_{2}$ are the frequency values of lasers under measurement obtained with CEM2 and frequency comb respectively. $U_{1}$ and $U_{2}$ are expanded uncertainties $(k=2)$ of lasers under measurement using a CEM2 and frequency comb respectively.

We obtained $C$ values less than 0.34 , this result indicates the compatibility of these measurements using two different standards (CEM2 and frequency comb).

Table 1 shows a summary of these measurements' results.

\section{Evaluating measurement uncertainty}

Estimating measurement uncertainty via studying the repeatability of the measurement is a well-established technique often used in dimensional metrology at many National Metrology Institutes. However, it often requires years of data to truly sample all sources of error in a typical dimensional measurement system.

Every GUM[13] compliant uncertainty calculation must start with the definition of the measurand and the formulation of the model equation for it. In frequency metrology, special mathematical tools have been developed to deal with time series of correlated data. In addition, the

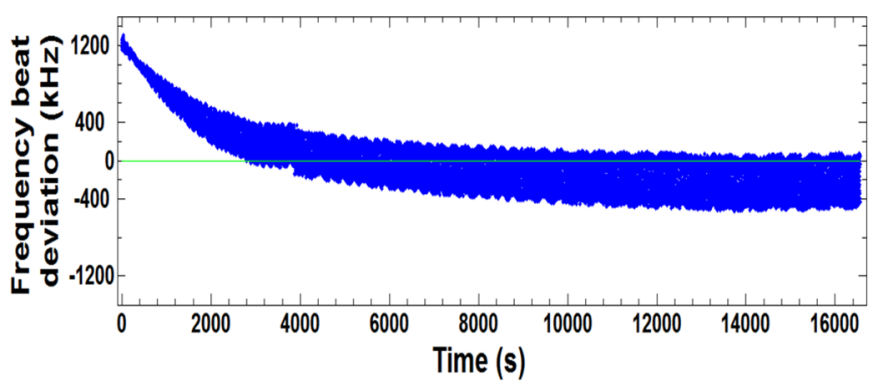

Fig. 6. $f_{\text {beat }}$ deviation of HP5517D laser against frequency comb.

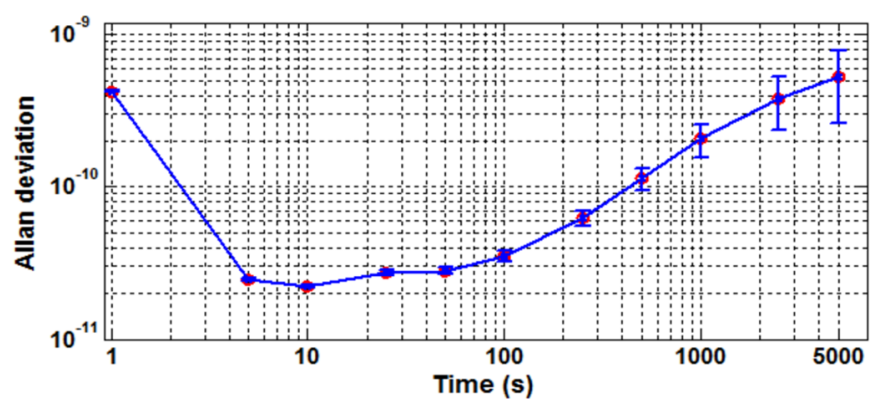

Fig. 8. Allan deviation of $f_{\text {beat }}$ note between HP5517D and femtosecond comb. Error bars corresponding to the standard uncertainty are shown for longer averaging times, where the error is large enough to be significant.

use of relative uncertainties is common practice in this field. However, we stay at a more traditional treatment [14]; we start with the comb equation (6) which relates the laser frequency (the measurand) to the repetition, offset frequencies of the comb, and the beat frequency with the $n$th comb mode, respectively.

$$
f_{c w}=n f_{r} \pm 2 \bar{f}_{0} \pm \bar{f}_{\text {beat }},
$$

where $\bar{f}_{0}$ and $\bar{f}_{\text {beat }}$ are calculated as the mean value of the offset and beat frequencies found in the measurement process, then

$$
\bar{f}_{0}=\left(\sum_{i} f_{i}\right) / m ; \bar{f}_{\text {beat }}=\left(\sum_{j} f_{j}\right) / m .
$$

Applying the law of propagation of uncertainty, the corresponding expression is obtained by using combined standard uncertainty $u_{c}(y)$ (assuming the absence of correlations).

$$
\begin{aligned}
u_{c}^{2}(y) & =\sum_{i=1}^{N}\left[\frac{\partial f}{\partial x_{i}}\right]^{2} u^{2}\left(x_{i}\right)=\sum_{i=1}^{N}\left[c_{i} u\left(x_{i}\right)\right]^{2} \\
& =\sum_{i=1}^{N} u_{i}^{2}(y) .
\end{aligned}
$$

In our case,

$$
u^{2}\left(f_{c w}\right)=2^{2} u^{2}\left(\bar{f}_{0}\right)+n^{2} u^{2}\left(f_{r}\right)+u^{2}\left(\bar{f}_{\text {beat }}\right) .
$$


Table 1. Frequencies of lasers under calibration measured by CEM2 and frequency comb printed as a difference from nominal frequency values. All values in $\mathrm{MHz}$.

\begin{tabular}{llll}
\hline Type of lasers & $\begin{array}{l}\text { Nominal } \\
\text { frequency }\end{array}$ & $\begin{array}{l}\text { Deviation from } \\
\text { nominal frequency } \\
\text { (with CEM2) }\end{array}$ & $\begin{array}{l}\text { Deviation from nominal frequency } \\
\text { (with comb) }\end{array}$ \\
\hline HP5517D & 473612254 & $22.76 \pm 1.2$ & $22.79 \pm 1.0$ \\
HP5519A & 473612254 & $19.40 \pm 9.5$ & $19.10 \pm 0.9$ \\
TESA-RSD/SR & 473612750 & $9.99 \pm 0.5$ & $9.98 \pm 0.3$ \\
\hline
\end{tabular}

Table 2. The expanded uncertainty sources values involving in the measurement of frequency laser.

\begin{tabular}{ll}
\hline Uncertainty budget & $U(k=2)$ \\
\hline$u_{\sigma}($ comb stability $)$ & $1.9 \times 10^{-13}$ \\
$u_{C s}(24 \mathrm{~h})$ & $0.3 \times 10^{-13}$ \\
$u_{\text {comb }}$ & $2.0 \times 10^{-13}$ \\
$u_{\text {beat }}(\mathrm{Tesa} \mathrm{RSD} / \mathrm{SR})$ & $6.1 \times 10^{-10}$ \\
$u_{\text {beat }}(\mathrm{HP} 5517 \mathrm{D})$ & $2.1 \times 10^{-09}$ \\
$u_{\text {beat }}(\mathrm{HP} 5519 \mathrm{~A})$ & $2.2 \times 10^{-09}$ \\
\hline
\end{tabular}

By collecting everything and substituting, we end up with the model equation for a laser calibration using a comb

$$
u^{2}\left(f_{c w}\right)=u^{2}(c o m b)+u^{2}\left(\bar{f}_{\text {beat }}\right) .
$$

Next we analyse each of the uncertainty contributions.

\subsection{Uncertainty associated to the reference standard (frequency comb)}

The uncertainty of the frequency comb depends on the uncertainty in determining the offset frequency, $\bar{f}_{0}$ and the repetition frequency, $f_{r}$. However, both frequencies depend on two common contributions due to the stability of the synthesizer, and to the time reference that is given by the Cs atomic clock.

The uncertainty of the parameters that define the frequency comb is included within the stability of the frequency comb, characterized by the Allan variance, which is the internal counter of the comb synthesizer. Using the Allan variance as a contribution to the uncertainty avoids the correlations because the stability considers the direct contribution of servos, PLLs (Phase Locked Loops) and system electronics. For an integration time of $1000 \mathrm{~s}$, the Allan deviation is $u_{\sigma}=9.5 \times 10^{-14}[8]$. In each calibration performed it has been verified that the stability value is less than or equal to $u_{\sigma}$. Furthermore, the frequency comb is referenced to a primary frequency standard, a $C s$ atomic clock integrated into the network of atomic clocks of the Navy Observatory, ROA

$$
u_{\text {clock }}^{2}=u_{C s}^{2} .
$$

The certificate value emitted by ROA, which is for $24 \mathrm{~h}$, is $u_{C s}=0.32 \times 10^{-13}$. Finally, the uncertainty of the reference standard frequency comb is, $U(k=2)=2 \times 10^{-13}$.

\subsection{Uncertainty due to beat contribution}

The uncertainty associated with the measured value of the beat signal is estimated using a type B evaluation of uncertainty. To do so, we consider a type of rectangular distribution, with an amplitude $\left(f_{\max }-f_{\min }\right)$, where the first is the maximum value and the second is the minimum one of the frequencies found

$$
u\left(\bar{f}_{\text {beat }}\right)=\frac{\left(\frac{f_{\max }-f_{\min }}{\sqrt{12}}\right)}{f_{c w}} .
$$

Assigning a number of degrees of freedom $v_{i}=100$, this is a conservative approach established in Length Area of CEM by which the rectangular distributions are associated with an effective number of degrees of freedom equal to 100 , to avoid an "infinite" number of degrees, which would suppose a total security as regard to the limit's values of distribution. Therefore, equation (14) is written as

$$
u^{2}\left(f_{c w}\right)=\left(u_{\sigma}\right)^{2}+\left(u_{C s} / 2\right)^{2}+\left(\frac{\left(\frac{f_{\max }-f_{\min }}{\sqrt{12}}\right)}{f_{c w}}\right)^{2} .
$$

The expanded uncertainty, $U$ is obtained by multiplying the combined standard uncertainty $u\left(f_{c w}\right)$ by a coverage factor $k$

$$
U=k \cdot u\left(f_{c w}\right),
$$

where $k$ is a function of the effective degrees of freedom obtained by Welch-Satterthwaite formula [13], from the values and the degrees of freedom of the partial uncertainties, for the desired confidence value, typically $95 \%$. If it can be assumed a normal distribution the factor $k=2$ is often used. In Table 2 we summarise the uncertainty budget.

\section{Conclusions}

Optical frequency combs have received much attention in recent years due to their enormous potential in optical frequency metrology applications, taking advantage of the 
continuous breakthroughs in the field of time and frequency metrology. The absolute frequency measurements of Zeeman-stabilized $\mathrm{He}-\mathrm{Ne}$ lasers with our femtosecond laser frequency comb are in good compatibility with previous studies using a CEM2 as primary standard. In addition, the stability of beat frequency of Tesa RSD/SD is dominated by the typical white noise; while the stability of beat frequency of HP5517D and HP5519A at longer averaging times is degraded and corresponds to the drift.

By using Allan variance analysis, we estimate a CMC of frequency comb in $2 \times 10^{-13}$. We evaluate the measurement uncertainty for comb-based laser frequency calibrations in accordance with GUM analysis. Finally, this realisation of metre based on femtosecond comb improves measurement accuracy more than 100 times compared to current method based on iodine-stabilized lasers.

A very serious contribution of anonymous referees to the improvement of this manuscript is gratefully acknowledged. Author wish also, to thank the Spanish CEM, and the Ministry of Industry, Energy and Tourism, MINETUR, for their support to this work in the period of 2009-2013.

\section{References}

1. International Committee of Poinds and Measures and the Consultative Committee for the Definition of the Meter, Documents concerning the new definition of the metre, Metrologia 19, 163-177 (1984)

2. T.J. Quinn, International report: Practical realization of the definition of the metre, including recommended radiations of other optical frequency standards (2001), Metrologia 40, 103-133 (2003)

3. T.J. Quinn, International report: Practical realization of the definition of the metre (1997), Metrologia 36, 211-244 (1999)
4. D.J. Jones, S.A. Diddams, J.K. Ranka, A. Stentz, R.S. Windeler, S.T. Cundiff, Carrier-envelope phase control of femtosecond mode-locked lasers and direct optical frequency synthesis, Science 288, 635-639 (2000)

5. T. Udem, R. Holzwarth, T.W. Hänsch, Optical frequency metrology, Nature 416, 233-237 (2002)

6. P. Balling, Laser frequency stabilization and measurement of optical frequencies: Development of acetylene-stabilized DFB laser diode at $1542 \mathrm{~nm}$ and absolute frequency measurement by femtosecond comb, Ph.D. thesis, Lambert Academic Publishing, 2009

7. S. Ferreira-Barragáns, M. Mar Pérez-Hernández, B. Samoudi, E. Prieto, Realisation of the metre by optical frequency comb: Applications in length metrology, Proc. SPIE 8001, 1-8 (2011)

8. B. Samoudi, M. Mar Pérez-Hernández, S. Ferreira-Barragáns, E. Prieto, Absolute optical frequency measurements on iodinestabilized $\mathrm{He}-\mathrm{Ne}$ at $633 \mathrm{~nm}$ by using a femtosecond laser frequency comb, Int. J. Metrol. Qual. Eng. 3, 101-106 (2012)

9. D.J. Jones, S.A. Diddams, J.K. Ranka, A. Stentz, R.S. Windeler, S.T. Cundiff, Carrier-envelope phase control of femtosecond mode-locked lasers and direct optical frequency synthesis, Science 288, 635-639 (2000)

10. R. Holzwarth, T. Udem, T.W. Hänsch, J.C. Knight, W.J. Wadsworth, P.St.J. Russell, Optical frequency synthesizer for precision spectroscopy, Phys. Rev. Lett. 85, 2264-2267 (2000)

11. W.J. Riley, Handbook of Frequency Stability Analysis, NIST Special Publications (U.S. Government Printing Office, Washington, 2008), p. 1065

12. D.W. Allan, Should the classical variance be used as a basic measure in standard metrology? IEEE Trans. Instrum. Meas. IM-36, 646-654 (1987)

13. BIPM, Evaluation of measurement data - Guide to the expression of uncertainty in measurement, JCGM 100 (2008)

14. M. Matus, P. Balling, W. Mache, A. Nießner, P. Křen, Uncertainty estimation for comb based laser calibration by direct comparison of 3 different combs, MacroScale (2011)

Cite this article as: Bousselham Samoudi, Realisation of the metre by using a femtosecond laser frequency comb: applications in optical frequency metrology, Int. J. Metrol. Qual. Eng. 8, 16 (2017) 